\title{
Abrasion dentaire et travail spécialisé dans la population natoufienne de Mallaha (Israël)
}

\author{
Fanny Bocquentin ${ }^{\mathrm{a}, *}$, Pascal Sellier ${ }^{\mathrm{a}}$, Pascal Murail ${ }^{\mathrm{a}}$ \\ a Laboratoire d'anthropologie des populations du passé, UMR 5199-PACEA, université Bordeaux-1, 33405 Talence cedex, France
}

\section{Résumé}

Sur l'ensemble de la population de l'Épipaléolithique levantin (13000-9500 av. J.-C.) examinée, deux individus, qui appartiennent à la phase ancienne de l'occupation du site de Mallaha, témoignent d'une utilisation intensive de leurs dents comme outils. Bien que cette pratique ait souvent été suggérée pour des populations plus anciennes, il s'agit ici du plus ancien cas décrit qui soit incontestable. La morphologie de cette usure dentaire est étudiée dans une perspective fonctionnelle et des hypothèses sont proposées sur la nature du travail effectué. Une répartition des tâches au sein des chaînes opératoires semble acquise. Pour citer cet article : F. Bocquentin et al., C. R. Palevol 4 (2005).

(C) 2005 Académie des sciences. Publié par Elsevier SAS. Tous droits réservés.

\begin{abstract}
Tooth abrasion and task activity in the Natufian population of Mallaha (Israel). Of the Levantine Epipalaeolithic population studied (13000-9500 cal BC), two individuals belonging to the early phase of occupation of the Mallaha site reveal intensive use of their teeth as tools. Although it was often suggested that ancient populations had such practice, we described in this study the oldest undeniable case. The morphology of this dental wear is studied from a functional point of view and hypotheses are put forward as to the type of work carried out. A sharing of tasks within the production chaînes opératoires appears to be established. To cite this article: F. Bocquentin et al., C. R. Palevol 4 (2005).
\end{abstract}

(C) 2005 Académie des sciences. Publié par Elsevier SAS. Tous droits réservés.

Mots-clés : Natoufien ; Dents outils ; Abrasion occlusale ; Stries ; Israël

Keywords: Natufian; Tooth use; Occlusal abrasion; Microwear; Israel

\footnotetext{
* Auteur correspondant.

Adresse e-mail : f.bocquentin@anthropologie.u-bordeaux1.fr (F. Bocquentin).
} 
«Le premier et le plus naturel objet technique, et en même temps moyen technique de l'homme, c'est son corps. »

Marcel Mauss, 1936 [20]

\section{Introduction}

Malgré une certaine réticence sociale, il n'est pas rare aujourd'hui que nous utilisions nos dents, les incisives en particulier, pour couper ou maintenir un objet. Les sociétés du passé faisaient une utilisation des dents bien plus intensive que la nôtre et l'ethnologie a parfois décrit la denture comme une véritable troisième main [23,26]. Davantage encore, la denture peut être utilisée comme un outil spécifique où le rôle mécanique des dents ainsi que le rôle chimique de la salive sont mis à profit pour transformer les qualités d'un matériau et modifier sa forme. Ce travail, lorsqu'il est continu et effectué par des gestes répétitifs, laisse sur les dents des traces qui se distinguent d'une usure normale due à la mastication. Nous avons cherché à reconnaître une telle utilisation spécialisée des dents dans la population natoufienne qui a occupé le Sud-Ouest du Levant à la fin de l'Épipaléolithique entre 13000 et 9500 av. J.-C [2].

\section{Matériel étudié et contexte archéologique}

Le corpus étudié compte 358 individus, qui représentent la quasi-totalité de cette population natoufienne mise au jour. Nous avons répertorié 18 individus, parmi les 235 dentures conservées fonctionnelles (représentées par 319 dents déciduales et 3191 dents permanentes), pour lesquels l'usure dentaire se distingue d'une usure normale [4]. Ces individus sont issus de différents groupes ; leur répartition par sexe est comparable à la répartition globale. Le plus jeune sujet est un adolescent. Dans la majorité des cas, les stigmates observés sont peu marqués et témoignent d'une activité non alimentaire exceptionnelle ou débutante. Deux individus, cependant, montrent une utilisation fréquente de leurs dents dans des activités que l'on va chercher à définir. Ces individus proviennent tous deux de l'occupation la plus ancienne de Mallaha (11700 et 11300 ans BP) [32], un site majeur dont l'occupation a perduré pendant plus de 1500 ans. Ils étaient inhumés

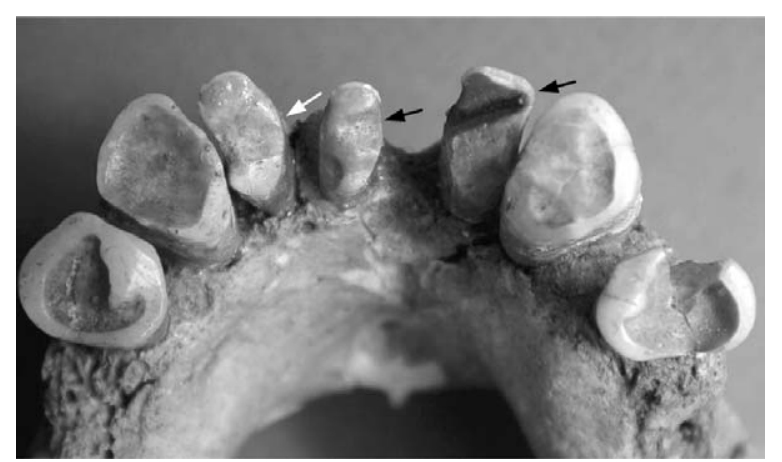

Fig. 1. H92 : Partie antérieure de la mandibule, vue supérieure. Les flèches indiquent la position et l'orientation des sillons.

Fig. 1. H92: Anterior part of the mandible, superior aspect. Position and orientation of the grooves are shown by arrows.

à proximité l'un de l'autre, dans le secteur de l'abri 131, et reposaient sur leur côté droit, les genoux fléchis et la main gauche ramenée contre le front [28].

\section{Une abrasion dentaire exceptionnelle}

Ces deux sujets, de sexe masculin [4], sont parmi les plus éprouvés de la population d'un point de vue articulaire ; l'un d'eux (H98) est atteint d'une maladie hyperostosique (DISH), qui confirme qu'il s'agit d'un individu âgé. Le fort degré d'usure dentaire ainsi que la fréquence des pertes ante mortem de ces deux individus sont comparables aux autres individus âgés de la même population, mais l'usure par abrasion que nous allons décrire leur est spécifique.

H92 a perdu de nombreuses dents avant sa mort, essentiellement des dents postérieures. Le degré d'usure est particulièrement remarquable sur les incisives inférieures, pour lesquelles seul un mince liseré d'émail subsiste sur le bord vestibulaire. Les racines, recouvertes de dentine secondaire, font office de surfaces occlusales et chacune d'entres elles est creusée par un sillon transversal (Fig. 1). Les bords de ces sillons ne sont pas parallèles, mais s'ouvrent, et parfois débordent sur la face mésiale. Systématiquement, en arrière du sillon, on observe une surface polie qui s'étend jusqu' au parodonte, en conservant une obliquité proche ou identique à celle du sillon lui-même. Dans le cas des incisives latérales, cette surface polie déborde sur les canines adjacentes qui, par ailleurs, ne présentent pas d'autres stigmates remarquables. Les prémolaires ne présentent rien de particulier non plus, ni les molaires, à l'excep- 


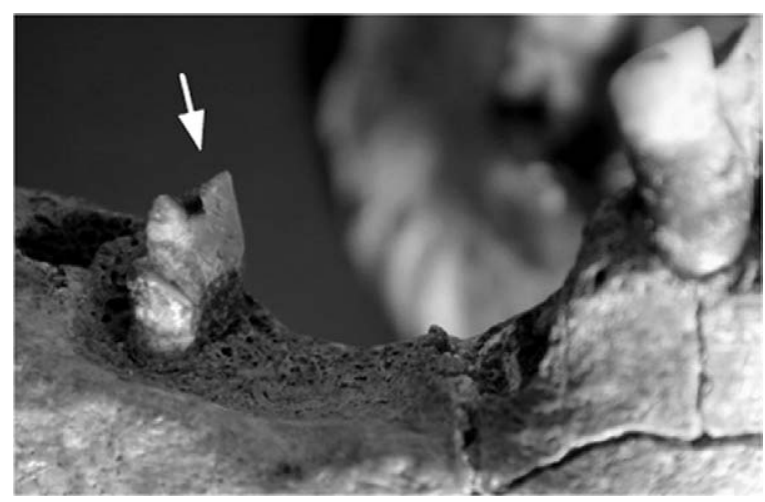

Fig. 2. H92 : Partie droite du corps de la mandibule entre la première prémolaire et la racine mésiale de la seconde molaire (vue latérale droite). La flèche indique la présence d'une surface abrasée perpendiculairement au plan masticatoire.

Fig. 2. H92: Part of the right mandibular body between the first premolar and the mesial root of the second molar (right lateral aspect). The arrow is pointing at an abraded surface perpendicular to the occlusal plane.

tion d'un petit fragment conservé de racine de la seconde molaire droite, dont la face mésiale est abrasée perpendiculairement au plan masticatoire (Fig. 2). Les incisives du maxillaire, contrairement à ce que l'on pourrait attendre, ne montrent pas d'usure complémentaire aux sillons inférieurs. Seule la deuxième molaire droite montre un poli très net, facetté et oblique à $45^{\circ}$ par rapport au plan occlusal (Fig. 3).

Sur la mandibule de H98 ne subsistent que les racines des deux canines, des deux premières prémolaires et de la deuxième prémolaire droite ; les autres dents sont tombées avant la mort (Fig. 4). Les surfaces occlu-

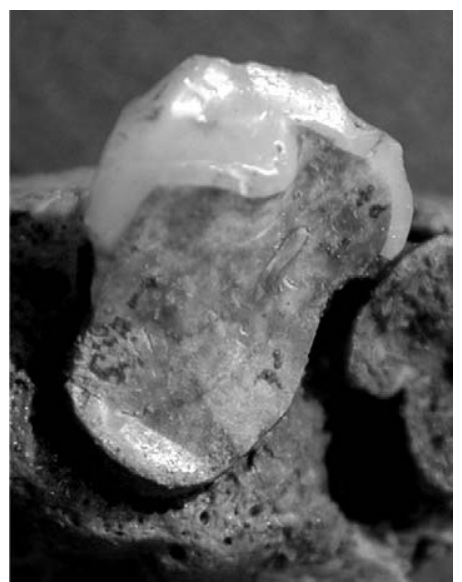

Fig. 3. H92 : Deuxième molaire supérieure droite, vue inférieure. Fig. 3. H92: Right upper second molar, inferior aspect.

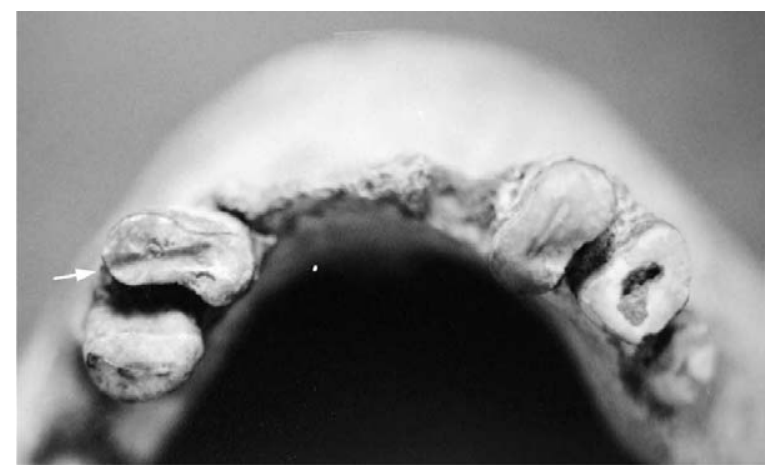

Fig. 4. H98 : Partie antérieure de la mandibule, vue supérieure. Les flèches indiquent la position et l'orientation des sillons.

Fig. 4. H98: Anterior part of the mandible, superior aspect. Position and orientation of the grooves are shown by arrows.

sales sont arrondies et celles des canines sont parcourues par des sillons transversaux, moins obliques que sur les incisives de H92. Les bords de ces sillons, une fois de plus, ne sont pas parallèles et leurs extrémités se poursuivent légèrement sur les faces adjacentes. À l'intérieur de ces deux sillons, la dentine secondaire est fortement lustrée. On observe sur le maxillaire des stigmates strictement complémentaires de ceux qui ont été observés sur la mandibule, ce qui témoigne d'une utilisation des dents en occlusion, la bouche fermée (Fig. 5). La première molaire droite présente, quant à elle, une très grande surface polie, comparable à celle de H92, qui descend depuis le plan occlusal de la couronne jusqu'aux deux tiers de la racine mise à nu (Fig. 6). Ce poli déborde sur la racine de la molaire voisine. De façon comparable, l'abrasion a transformé

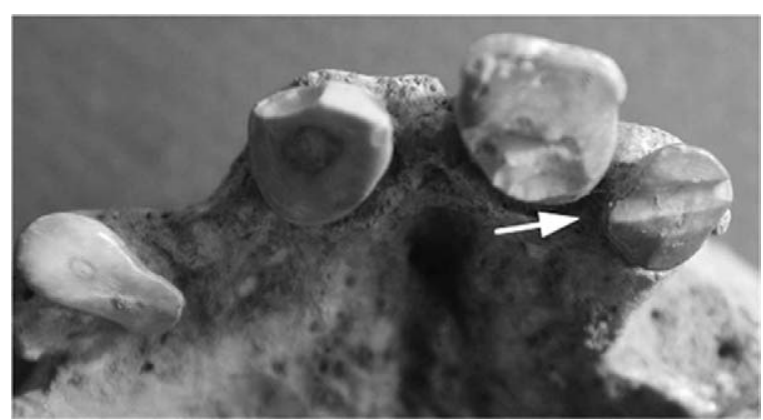

Fig. 5. H98 : Partie antérieure du maxillaire, vue inférieure. La flèche indique la position et l'orientation d'un sillon qui vient compléter, en occlusion, celui observé sur la canine inférieure gauche.

Fig. 5. H98: Anterior part of the maxillae, inferior aspect. The arrow is pointing at the position and orientation of the groove which is a continuation of the groove on the lower left canine (when the teeth are in occlusal position). 


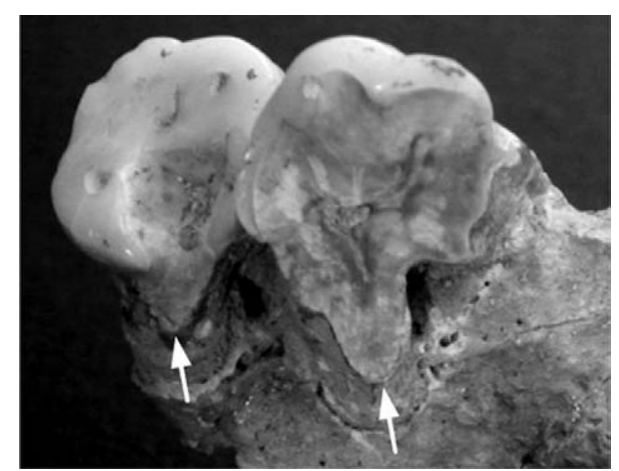

Fig. 6. H98 : Première et deuxième molaires supérieures droites, vue linguale. Les flèches indiquent le plan d'abrasion.

Fig. 6. H98: Right upper first and second molars, lingual aspect. The arrows are pointing at the abrasion plane.

le bord incisif des dents antérieures en un plateau occlusal, fortement incliné vers la face vestibulaire. Cette inclinaison est d'autant plus marquée que les dents ellesmêmes ont aussi basculé vers l'extérieur.

\section{L'utilisation des dents comme outils}

L'usure dentaire de ces deux individus se distingue clairement d'une usure due à la mastication par la présence des sillons et du facettage des surfaces occlusales selon des orientations incompatibles avec le plan masticatoire présumé, quel que soit le régime alimentaire ou les facteurs abrasifs du milieu environnant [29]. Cette usure artificielle n'est pas uniforme, mais nous observons plusieurs caractéristiques récurrentes : les sillons se situent exclusivement sur les dents antérieures et sont associés à un polissage de la dent. Ce polissage peut être convexe et couvrir la totalité de la dent, ou bien il peut être parfaitement plat et ne concerner qu'une partie de la surface occlusale, en général, celle qui se trouve en arrière du sillon. Ce type de polissage plat et oblique est aussi observé en l'absence de sillons sur les incisives de H98. En ce qui concerne les dents postérieures, le polissage est toujours abrupt et forme un angle proche de $90^{\circ}$ avec le plan occlusal ; il envahit la totalité de la face linguale ou de la face mésiale de la dent.

Des sillons comparables n'ont été qu'exceptionnellement publiés et toujours pour des populations plus récentes $[3,7,14,30]$. Plus proche du contexte natoufien, des sillons similaires ont aussi été mentionnés pour la population néolithique du Sahara [24] ou pour celle de Abu Hureyra en Syrie [25]. Dans tous les cas, de tels stigmates sont interprétés comme le témoignage de l'utilisation des dents comme outils lors de la préparation de liens. L'hypothèse de liens en fibres végétales (tiges, écorces ou feuilles) a généralement la préférence des auteurs qui s'appuient sur des observations ethnologiques. Les dents sont utilisées pour diviser les fibres, éventuellement séparer l'écorce ou maintenir l'un des brins lors de l'opération de tressage [33]. Mais il pourrait également s'agir de fibres animales (tendons, boyaux, peau), pour lesquelles les dents antérieures sont aussi sollicitées lors du travail d'assouplissement et de calibrage dans un mouvement susceptible de laisser sur les dents des sillons par abrasion comparable $[6,18,21,27]$.

Le polissage fortement incliné des dents supérieures est en revanche décrit plus fréquemment ; il est généralement attribué à la mastication des peaux (par exemple, [16,18,19,27]). Il a parfois été nommé par les anthropologues français le « signe du cordonnier » [15]. De sources ethno-anthropologiques, les dents antérieures sont sollicitées, soit lors de la préparation du cuir, soit tout à la fin de la chaîne opératoire, pour assouplir un objet déjà terminé $[16,18,23,27]$. Les peaux sont tirées entre les dents d'arrière en avant et vers le bas ; il en résulte un polissage très oblique des molaires et des incisives $[11,16,18]$. Une utilisation similaire des dents a aussi été décrite pour l'assouplissement des aliments séchés en vue de leur consommation ou pour l'écrasement de fibres végétales $[18,23,30]$.

\section{Matériau travaillé et gestes effectués : discussion}

Il apparaît ainsi difficile de distinguer, par une observation macroscopique, l'origine animale ou végétale du matériau travaillé par les dents antérieures et postérieures. En revanche, les caractéristiques microscopiques de cette abrasion donnent des informations supplémentaires. Les micro-stries que nous avons pu observer au microscope électronique à balayage permettent tout d'abord de confirmer l'origine mécanique de l'usure. On observe effectivement, sur toutes les surfaces abrasées, des stries parallèles les unes aux autres, dont l'origine chimique ou taphonomique est exclue (par exemple, [12]). Ce sont ces stries qui, par leur orientation et leurs caractéristiques (profondeur, lar- 

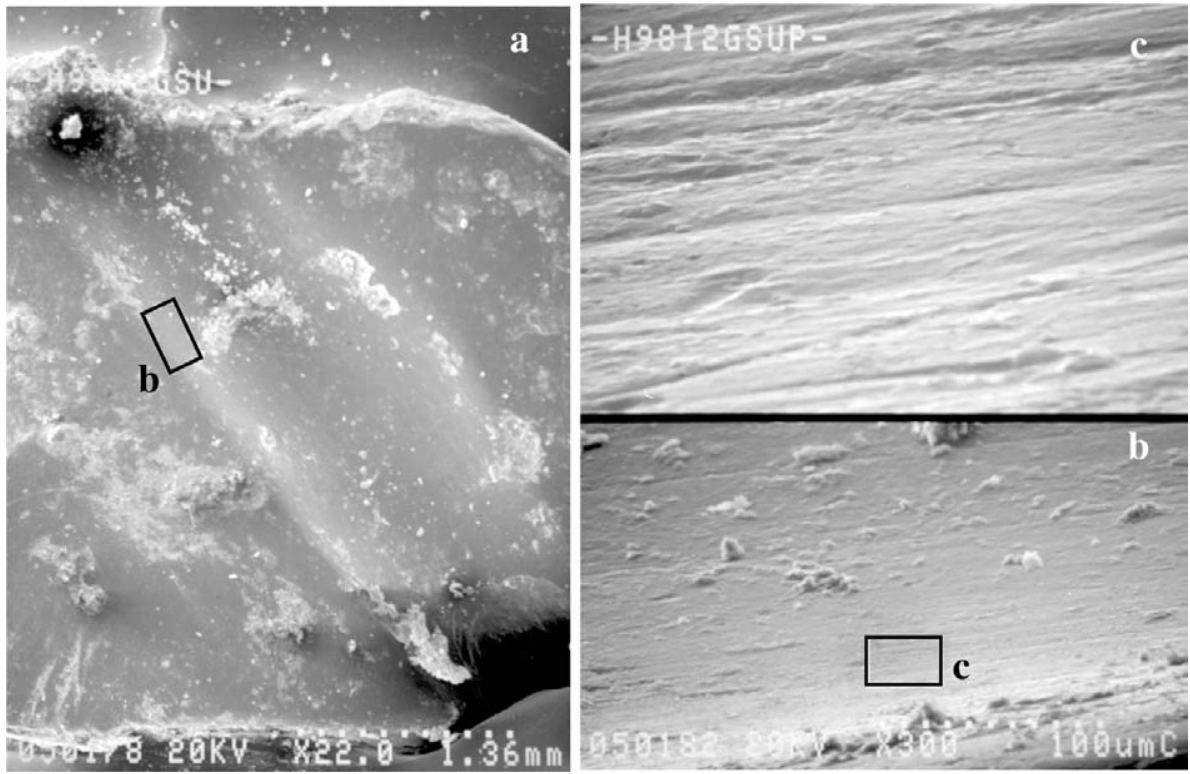

Fig. 7. H98 : Incisive latérale supérieure gauche : (a) grossissement de la face occlusale $(\times 22)$; (b) et (c) grossissement à l'intérieur du sillon $(\times 300$ et $\times 3000)$

Fig. 7. H98: Left upper lateral incisor. (a) Magnification $(\times 22)$ of the occlusal surface; $(\mathbf{b})$ and $(\mathbf{c})$ magnification $(\times 300$ and $\times 3000)$ inside the groove.

geur, forme, espacement et fréquence), permettent de discuter d'une part, des mouvements qui ont été donnés à l'objet et d'autre part, la nature de cet objet (par exemple, $[1,10])$.

Les études archéologiques en tracéologie livrent des informations sur la formation et la spécificité des usures en fonction du matériau travaillé. Mais cette approche n'est pas entièrement satisfaisante pour interpréter les traces que nous observons, car les paramètres étudiés sont différents. Par ailleurs, rares sont les observations faites sur des stigmates microscopiques laissés par le frottement d'un objet sur la dentine [29] ; ces stigmates se distinguent cependant nettement de ceux laissés par le même objet sur l'émail, qui est beaucoup plus résistant [9].

Néanmoins, de toutes les dents artificiellement abrasées que nous avons pu observer au MEB, une certaine homogénéité se dégage entre les stigmates et permet de proposer des hypothèses sur les gestes et le matériau travaillé. En ce qui concerne les dents antérieures, seuls les sillons de H98 ont pu être observés au microscope. Les stries y sont nombreuses et s'orientent parallèlement au grand axe du sillon (Fig. 7). Sur la surface polie en arrière du sillon, les stries s'orientent perpendiculairement à cet axe. Les caractéristiques quantita- tives et qualitatives de ces stries photographiées sur la dentine secondaire se rapprochent davantage de celles qui sont laissées par le travail de fibres végétales. Il semble, en effet que l'abrasion obtenue par frottement de fibres animales, des tendons en particulier, se caractérise par des stigmates microscopiques différents avec des stries courtes et larges [5]. Par ailleurs, le lustre très marqué qui a été observé sur certaines de ces dents antérieures est également en faveur d'un matériau fortement abrasif (plantes) ou d'une addition de particules abrasives au matériau travaillé $[5,22]$. Ceci, selon les observations faites chez différentes populations, ne semble pas être le cas pour l'assouplissement des tendons $[6,27]$.

En ce qui concerne les molaires supérieures, le mouvement est clairement orienté du haut vers le bas et vers l'avant pour les deux individus. Les stries laissées par cet étirement du matériau sont nombreuses, très fines et régulières (Fig. 8). Elles plaident plutôt en faveur du travail de fibres végétales, fibres riches en silice et donc plus abrasives que d'autres matériaux (par exemple, $[13,31])$.

Finalement, l'étude macroscopique et microscopique confirme bien l'utilisation des dents comme outils dans une opération de transformation d'un matériau 

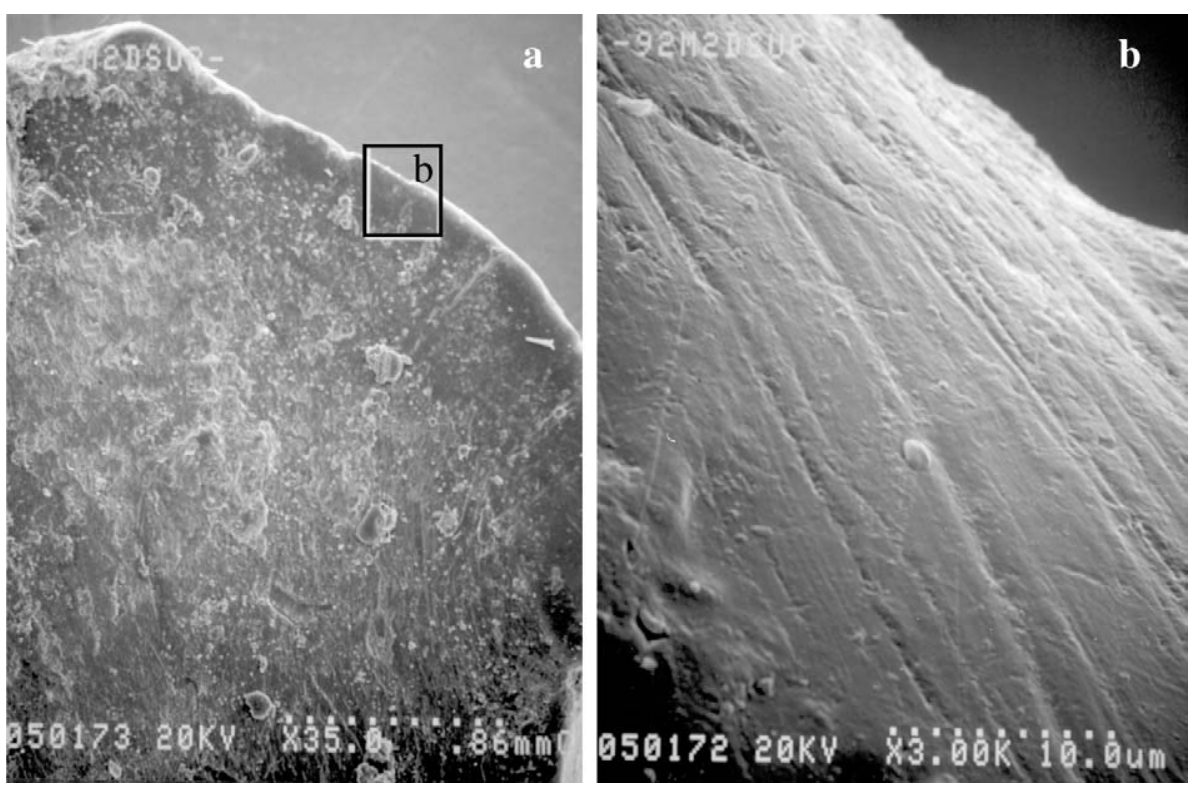

Fig. 8. H92 : Deuxième molaire supérieure droite : (a) grossissement du bord vestibulo-mésial $(\times 35)$; (b) grossissement de l'émail $(\times 3000)$. Fig. 8. H92: Right upper second molar. (a) Magnification of the vestibulo-lingual edge $(\times 35)$; (b) Magnification of the enamel $(\times 3000)$.

souple, plus probablement d'origine végétale. Les traces laissées sur les dents témoignent de gestes récurrents intégrés dans un processus opératoire fixe. Cependant, ces gestes ont dû se modifier au fur et à mesure de l'évolution de l'arcade dentaire et il est probable, en particulier, que les prémolaires, majoritairement perdues avant la mort, jouaient un rôle important dans cette technique. L'association des polis et des sillons suggère que le mouvement donné aux objets n'était pas unique et que sa transformation impliquait sans doute un enchaînement de gestes complémentaires.

L'environnement de Mallaha, qui se situe à quelques centaines de mètres du grand lac Houleh, autorise l'hypothèse du travail de fibres végétales. En effet, l'analyse pollinique a mis en évidence, dans les niveaux anciens de Mallaha, l'importance des plantes aquatiques, identiques à celles qui poussent aujourd'hui, ainsi que la présence du saule [17]. Il s'agit de plantes solides et flexibles typiquement utilisées pour la vannerie encore aujourd'hui.

\section{Conclusion}

Il apparaît que ces deux individus utilisaient leurs dents plus fréquemment que le reste de la population natoufienne, et pour une tâche spécifique. C'est peut- être la présence du lac à proximité du site qui fait de Mallaha un cas particulier. Non seulement il fournissait les plantes adéquates à la préparation de liens, mais encore l'exploitation de ses ressources a probablement encouragé leur fabrication, notamment pour des filets de pêche. L'ichtyofaune est, en effet, présente en quantité sur le site [8]. Par ailleurs, le Natoufien ancien de Mallaha témoigne d'un fort degré de sédentarité plus propice qu'ailleurs au développement d'activités spécialisées. Et il semble effectivement que le travail de transformation des fibres soit une activité réservée à un petit nombre d'individus, qui jouent un rôle spécifique au sein de la communauté de Mallaha ou qui l'acquièrent par cette activité. Cette division du travail est remarquable dans ce contexte natoufien où le système économique et social est en cours de transformations majeures ; elle rapproche ces chasseurs/cueilleurs sédentaires épipaléolithiques des sociétés néolithiques qui vont suivre, où une répartition comparable des tâches a également été proposée [25].

\section{Remerciements}

Cette étude a été financée par le programme Training and Mobility of Researchers de la Communauté européenne. Le matériel a pu être étudié grâce à l'aima- 
ble autorisation de B. Arensburg et I. Hershkovitz (université Sackler de médecine, Tel-Aviv). Nous souhaitons aussi remercier F. Rouais, E. Pubert, H. Khalaily, T. Molleson, F. d'Errico et C.S. Larsen pour leur aide.

\section{Références}

[1] L.R. Backwell, F. D’Errico, Evidence of termite foraging by swartkrans early hominids, Proc. Natl Acad. Sci. USA 98 (2001) 1358-1363.

[2] O. Bar-Yosef, F. Valla, The Natufian culture in the Levant, Int. Monogr. Prehist. (Archaeol. Ser., 1), Ann Arbor, 1991.

[3] M. Billard, L'abrasion artificielle des dents comme aspect révélateur d'habitudes culturelles : observation comparée de deux sites du Chalcolithique-Bronze ancien (Drôme et Ardèche, in: L. Buchet (Ed.), L'identité des populations archéologiques, Sophia-Antipolis, APDCA, 1996, pp. 330 343.

[4] F. Bocquentin, Pratiques funéraires, paramètres biologiques et identités culturelles au Natoufien : une analyse archéoanthropologique, thèse, université Bordeaux-1, Talence, http: $/ / 147.210 .235 .3 /$ proprietes.html?numero ordre $=2769$.

[5] L. Bouchneb, B. Maureille, Sillons d'usure interproximaux sur les dents : reproduction expérimentale, analyse et application des résultats aux observations sur la lignée Néandertalienne, in: Bull. Mém. Soc. Anthropol. Paris. 16 n. s, 2004, pp. 37-48.

[6] T. Brown, S. Molnar, Interproximal Grooving and Task Activity in Australia, Am. J. Phys. Anthropol. 81 (1990) 545-553.

[7] J.S. Cybulski, Tooth wear and material culture: precontact patterns in the Tsimshian area, British Colombia, Syesis 7 (1974) 31-35.

[8] J. Desse, L'ichtyofaune, in: J. Bouchud (Ed.), La faune du gisement natoufien de Mallaha (Eynan), Israël, Mém. Trav. Centre Rech. Fr. Jérus., vol. 4, Association Paléorient, Paris, 1987, pp. 151-156.

[9] F. D'Errico, G. Villa, G. Fornaciari, Dental esthetics of an Italian Renaissance noblewoman, Isabella d'Aragona. A case of chronic mercury intoxication, Ossa 13 (1998) 233-254.

[10] K.R. Gordon, Microfracture patterns of abrasive wear striations on teeth indicate directionality, Am. J. Phys. Anthropol. 63 (1984) 315-322.

[11] R.G. Hinton, Form and patterning of anterior tooth wear among aboriginal human groups, Am. J. Phys. Anthropol. 54 (1981) 555-564.

[12] T. King, P. Andrews, B. Boz, Effect of taphonomic processes on dental microwear, Am. J. Phys. Anthropol. 108 (1999) 359-373.

[13] C. Lalueza Fox, J. Juan, R. Albert, Phytolith Analysis on Dental calculus, enamel surface and burial soil: information about diet and paleoenvironment, Am. J. Phys. Anthropol. 101 (1996) 101-113.
[14] C.S. Larsen, Dental modifications and tool-use in the western Great Basin, Am. J. Phys. Anthropol. 67 (1985) 393-402.

[15] J. Lefèvre, Étude odontologique des hommes de Muge, Bull. Mem. Soc. Anthropol. Paris 10 (1973) 301-333.

[16] R.W. Leigh, Dental pathology of the Eskimo, Dent. Cos. 67 (1925) 884-898.

[17] A. Leroi-Gourhan, L'environnement de Mallaha (Eynan) au Natoufien, Paléorient 10 (1984) 103-105.

[18] G.F. Lous, 1970, The masticatory system used as tool, Dent. Abstr. 15 (1970) 457-458.

[19] J.R. Lukacs, R.F. Pastor, Activity induced patterns of dental abrasion in prehistoric Pakistan: Evidence from Mehrgarh and Harappa, Am. J. Phys. Anthropol. 76 (1988) 377-398.

[20] M. Mauss, Les techniques du corps, J. Psychol. 32 (1936) 271-293.

[21] E. Martin, Hadzabe, dernier peuple archer d'Afrique, Archers 16 (2001) 12-17.

[22] M.E. Mansur-Franchomme, Scanning electron microscopy of dry hide working tools: the role of abrasives and humidity in microwear polish formation, J. Archaeol. Sci. 10 (1983) 223230.

[23] C.F. Merbs, Patterns of activity-induced pathology in a Canadian Iniut population, National Museum of Man, Mercury Series, Archaeol. Surv. Can. 119, Ottawa, 1983.

[24] S. Minozzi, G. Manzi, F. Ricci, S. Di Lernia, S.M. Borgognini Tarli, Nonalimentary tooth use in Prehistory: an example from Early Holocene in central Sahara (Uan Muhuggiag, Tadrart Acasus, Lybia), Am. J. Phys. Anthropol. 120 (2003) 225-232.

[25] T. Molleson, The Eloquent Bones of Abu Hureyra, Sci, Am. 171 (1994) 70-75.

[26] S. Molnar, Tooth wear and culture: a survey of tooth functions among some prehistoric populations, Curr. Anthropol. 13 (1972) 511-526.

[27] P.O. Pedersen, Some dental aspects of anthropology, Dent. Rec. 72 (1952) 170-178.

[28] J. Perrot, D. Ladiray, O. Solivères-Masséi, Les hommes de Mallaha, (Eynan), Israël, Mém. Trav. Centre Rech. Fr. Jérus., Paris, 1988.

[29] A.S. Ryan, D.C. Johanson, Anterior dental microwear in Australopithecus afarensis: Comparisons with human and nonhuman primates, J. Hum. Evol. 18 (1989) 235-268.

[30] P.D. Schulz, Task activity and anterior tooth grooving in prehistoric California Indians, Am. J. Phys. Anthropol. 46 (1977) 87-92.

[31] P.S. Ungar, M.A. Spencer, Incisor microwear, diet and tooth use in three Amerindian populations, Am. J. Phys. Anthropol. 109 (1999) 387-396.

[32] F.R. Valla, Natufian Seasonality: a guess, in: T.R. Rocek, O. Bar-Yosef (Eds.), Seasonality and sedentism: archaeological perspectives from Old and New World sites, Harvard University Press, Harvard, 1998, pp. 93-108.

[33] M.M. Wheat, Survival Arts of the Primitive Paiutes, University of Nevada Press, Reno, 1967. 